\title{
On the characteristics of fast neutrino flavor instabilities in three-dimensional core-collapse supernova models
}

\author{
Sajad Abbar@, ${ }^{1, *}$ Francesco Capozzi®, ${ }^{1,2, \dagger}$ Robert Glas@, ${ }^{3,4, \$}$ H.-Thomas Janka@ ${ }^{3, \S}$ and Irene Tamborra $\oplus^{5, \|}$ \\ ${ }^{1}$ Max Planck Institut für Physik (Werner-Heisenberg-Institut), \\ Föhringer Ring 6, 80805 München, Germany \\ ${ }^{2}$ Center for Neutrino Physics, Department of Physics, Virginia Tech, Blacksburg, Virginia 24061, USA \\ ${ }^{3}$ Max-Planck-Institut für Astrophysik, Karl-Schwarzschild-Straße 1, D-85748 Garching, Germany \\ ${ }^{4}$ Excellence Cluster ORIGINS, Boltzmannstr. 2, D-85748 Garching, Germany \\ ${ }^{5}$ Niels Bohr International Academy and DARK, Niels Bohr Institute, University of Copenhagen, \\ Blegdamsvej 17, 2100 Copenhagen, Denmark
}

(Received 18 December 2020; accepted 19 February 2021; published 23 March 2021)

\begin{abstract}
We assess the occurrence of fast neutrino flavor instabilities in two three-dimensional state-of-the-art core-collapse supernova simulations performed using a two-moment three-species neutrino transport scheme: one with an exploding $9 \mathrm{M}_{\odot}$ and one with a nonexploding $20 \mathrm{M}_{\odot}$ model. Apart from confirming the presence of fast instabilities occurring within the neutrino decoupling and the supernova pre-shock regions, we detect flavor instabilities in the post-shock region for the exploding model. These instabilities are likely to be scattering-induced. In addition, the failure in achieving a successful explosion in the heavier supernova model seems to seriously hinder the occurrence of fast instabilities in the post-shock region. This is a consequence of the large matter densities behind the stalled or retreating shock, which implies high neutrino scattering rates and thus more isotropic distributions of neutrinos and antineutrinos. Our findings suggest that the supernova model properties and the fate of the explosion can remarkably affect the occurrence of fast instabilities. Hence, a larger set of realistic hydrodynamical simulations of the stellar collapse is needed in order to make reliable predictions on the flavor conversion physics.
\end{abstract}

DOI: $10.1103 /$ PhysRevD.103.063033

\section{INTRODUCTION}

Massive stars can end their lives in the form of corecollapse supernova (CCSN) explosions, where a huge amount of energy $\left(\sim 3 \times 10^{53} \mathrm{ergs}\right)$ is released of which almost $99 \%$ is in the form of neutrinos of all flavors [1-3]. In this context, neutrino flavor evolution can be a collective phenomenon in which neutrinos propagating with different momenta change their flavor altogether thanks to the neutrino-neutrino interactions in the SN environment [4-6].

Neutrino flavor conversions are important to the physics of CCSNe. In particular, they can change the neutrino and antineutrino energy distributions and consequently their

\footnotetext{
*abbar@mppmu.mpg.de

†capozzi@mppmu.mpg.de

trglas@mpa-garching.mpg.de

\$thj@mpa-garching.mpg.de

tamborra@nbi.ku.dk
}

Published by the American Physical Society under the terms of the Creative Commons Attribution 4.0 International license. Further distribution of this work must maintain attribution to the author(s) and the published article's title, journal citation, and DOI. Open access publication funded by the Max Planck Society. scattering rates. This can, at least in principle, impact the CCSN dynamics and the nucleosynthesis of heavy elements in the SN environment [7]. Moreover, understanding collective neutrino oscillations is necessary for analyzing the neutrino signal from the next galactic CCSN and the upcoming measurements of the diffuse SN neutrino background [8-11].

It has been shown that neutrinos can experience fast flavor conversions in dense neutrino media [12-37]. In contrast to the traditional slow modes which occur on scales determined by the vacuum frequency $(\gtrsim \mathcal{O}(1) \mathrm{km}$ for a typical SN neutrino), fast modes occur on scales $\sim G_{\mathrm{F}}^{-1} n_{\nu}^{-1}$, where $G_{\mathrm{F}}$ is the Fermi constant and $n_{\nu}$ is the neutrino number density. Such scales can be as short as a few $\mathrm{cm}$ in the vicinity of the proto-neutron star (PNS). Although both fast and slow modes can be triggered below the shock (for the latter this has been recently shown to be possible through matter turbulence [38]), fast modes remain unique because in the deepest regions of the $\mathrm{SN}$ core they can develop on scales $\sim$ few $\mathrm{cm}$, which are much shorter than the average neutrino scattering length.

It is widely believed that a necessary (and probably sufficient) condition for the occurrence of fast flavor instabilities is that the angular distributions of $\nu_{e}$ and $\bar{\nu}_{e}$ 
cross each other $[15,17,18,21,23,39]$. To be more precise, the angular distribution of the neutrino electron lepton number (ELN) should change its sign at a specific angle, i.e., it should have a crossing (see Sec. III for details). The first assessments of the occurrence of ELN crossings in CCSNe were based on the analytical two-bulb model $[12,13,40,41]$ in which two separate neutrinospheres were considered for $\nu_{e}$ and $\bar{\nu}_{e}$. Although ELN crossings are always present in such models, at least as long as the neutrino-antineutrino asymmetry parameter

$$
\alpha=n_{\bar{\nu}_{e}} / n_{\nu_{e}},
$$

is not very small, it was soon realized that their existence in realistic CCSN simulations is not guaranteed. In particular, ELN crossings were not found in the post-shock region of one-dimensional (1D) CCSN simulations $[42,43]$. However, recently several studies have reported the existence of ELN crossings and their associated fast instabilities in multidimensional (multi-D) simulations [44-48]. Fast instabilities can indeed occur via different mechanisms in different $\mathrm{SN}$ regions:

(i) In the convective layer of the PNS, well below the neutrinospheres where the angular distributions of $\nu_{e}$ and $\bar{\nu}_{e}$ are still almost isotropic [45,47,48]. Such a possibility originates from the strong convective activity therein, which can generate large amplitude modulations in the spatial distributions of $\nu_{e}$ and $\bar{\nu}_{e}$ number densities. Hence, zones can exist there, for which $\alpha$ is arbitrarily close to one and no matter how isotropic are the distributions of $\nu_{e}$ and $\bar{\nu}_{e}$, ELN crossings can always occur. However and despite such an interesting possibility, the physical implications of this class of ELN crossings are not very clear due to the nearly equal distributions of neutrinos and antineutrinos of all flavors (with chemical potential close to zero), which means that neutrino oscillations cannot significantly modify the flavor content of the neutrino gas.

(ii) Within the neutrino decoupling region just above the PNS [44,46,47]. The existence of ELN crossings in this region can be explained as follows. Due to the neutron richness of the SN matter, $\nu_{e}$ 's decouple at larger radii than $\bar{\nu}_{e}$ 's. As a consequence, the angular distributions of $\bar{\nu}_{e}$ 's are in general more peaked in the forward direction than those of $\nu_{e}$ 's, thus increasing the chance for the occurrence of ELN crossings. Nevertheless, ELN crossings can still only occur in zones where $\alpha$ is relatively close to one and are suppressed otherwise $[44,49]$. This means that asymmetry in the neutrino emission, caused by the LESA (lepton-emission self-sustained asymmetry) phenomenon [50,51], plays an important role here (other effects leading to asymmetric neutrino emission were discussed, e.g., in Ref. [52]). Indeed the associated asymmetric lepton number emission implies that the distribution of $\alpha$ is not uniform above the PNS and there can exist zones for which $\alpha$ is relatively close to one. This increases the chance for the occurrence of ELN crossings in such SN zones. Needless to say, this type of ELN crossings should be then seriously suppressed if LESA is not strong enough.

(iii) In the preshock $\mathrm{SN}$ region. As first pointed out in Ref. [43], this type of ELN crossings is generated by neutrino backward scatterings off heavy nuclei. At a fixed energy, the scattering rate of neutrinos is larger than the one of antineutrinos. However, taking into account that the latter have a larger average energy, one finds that $\bar{\nu}_{e}$ has a larger cross section. This feature allows antineutrinos to be dominant in the backward direction and generate a backward ELN crossing despite the fact that neutrinos have an overall larger number density. This sort of ELN crossings can be more generic than the ELN crossings discussed previously. In spite of this, such fast instabilities cannot impact the SN (shock) dynamics and in addition, their corresponding growth rates could be very small due to the smaller $n_{\nu}$ in the SN pre-shock region.

Considering the discussion above, perhaps the fast instabilities occurring within the neutrino decoupling region have the most important physical implications. Here, a remark is in order. In almost all the state-of-theart 3D CCSN simulations the equations for the neutrino transport are solved in terms of a few angular moments of the neutrino phase space distribution. This is done to avoid the extremely demanding computational requirements of solving the Boltzmann equation. Such an approximation prevents us from having direct information on the underlying neutrino angular distributions. The only exception is the Ref. [53] in which a 3D CCSN simulation was performed with full Boltzmann neutrino transport, but only up to $20 \mathrm{~ms}$ after bounce and still with severe limitations of the numerical resolution (see, e.g., Refs. [52,54-57] for examples of CCSN simulations with full Boltzmann neutrino transport). Most of the previous studies of the occurrence of fast instabilities have been based on either 1D/2D SN models or post-processing calculations in which the full neutrino Boltzmann equation is solved for static SN density profiles derived from less fashionable CCSN simulations. One may wonder whether the results obtained in this context can be confirmed/ improved when the state-of-the-art 3D CCSN simulations are considered. In fact the only study adopting such models found the occurrence of fast instabilities only in the convective layer of the PNS [48].

Although a significant amount of information gets lost using the neutrino angular moments, it is still possible to extract some useful information regarding the occurrence 
of ELN crossings. In particular, it has been shown that ELN crossings are guaranteed to exist if the neutrino angular moments meet certain criteria, as discussed in Sec. III. In this paper, we assess the possibility of the occurrence of fast instabilities in the SN environment by studying two 3D CCSN simulations of a $9 \mathrm{M}_{\odot}$ and a $20 \mathrm{M}_{\odot}$ model, which were recently performed by the Garching group [51,58] (discussed briefly in Sec. II). As we discuss in Sec. IV, not only do we confirm the possibility of the occurrence of fast instabilities within the neutrino decoupling and the preshock SN regions, but also we bring up a new possibility for the occurrence of probably scattering-induced fast instabilities in the post-shock SN region. Moreover, we demonstrate that there can be an important connection between the fate of the explosion and the chance for the occurrence of fast instabilities in CCSNe, i.e., the occurrence of ELN crossings can be seriously hindered in nonexploding SN models with high mass accretion rates.

\section{SUPERNOVA MODELS}

Our study is based on two self-consistent 3D CCSN simulations carried out by the Garching group [51,58]: in the first simulation, a $9 \mathrm{M}_{\odot}$ model successfully exploded via the delayed neutrino-heating mechanism with the shock revival setting in at about $300 \mathrm{~ms}$ after the core bounce, whereas in the second one, a more massive progenitor star with $20 \mathrm{M}_{\odot}$ failed to explode. Both simulations were performed with the Aenus-Alcar code [59-61], which solves the hydrodynamics equations describing the time-dependent flow properties of the stellar plasma, and the equations of a velocity- and energy-dependent two-moment neutrino transport scheme. In this so-called "M1" scheme, the computationally expensive solution of the Boltzmann transport equation is circumvented by the approximation of evolving the first two angular moment equations of the Boltzmann transport equation. This implies that the transport solver does not compute the time-dependent neutrino phase-space distribution functions, but instead it tracks the evolution of the energy-dependent moments of neutrino energy density and neutrino energy-flux density.

While in the original paper [58] the authors varied both the neutrino transport approximation and the numerical grid resolution, we consider here only the fiducial models with fully multi-D (FMD) neutrino transport and with higher grid resolution, i.e., models "s9.0 FMD H" and "s20 FMD H" for the $9 \mathrm{M}_{\odot}$ and $20 \mathrm{M}_{\odot}$ progenitor stars, respectively. These models were simulated in spherical polar coordinates $r$ (radius), $\Theta$ (polar angle), and $\Phi$ (azimuthal angle) with an effective angular resolution of roughly $2^{\circ}$, and with 640 radial zones spanning a range from 0 to $10^{4} \mathrm{~km}$.

In both simulations, the neutrino transport equations were solved for electron neutrinos $\left(\nu_{e}\right)$, electron antineutrinos $\left(\bar{\nu}_{e}\right)$, and a third species $\nu_{x}$ that represents all four kinds of heavy-lepton neutrinos $\left(\nu_{\mu}, \bar{\nu}_{\mu}, \nu_{\tau}\right.$, and $\left.\bar{\nu}_{\tau}\right)$, whose transport properties are considered to be identical. The interactions between neutrinos and the stellar matter included a whole set of absorption, emission, scattering, and thermal production processes, for which we refer the reader to the original publication.

\section{SEARCHING FOR FAST INSTABILITIES}

As mentioned above, a necessary condition for the existence of fast flavor instabilities in a dense neutrino gas is the presence of crossings in the ELN angular distribution. The latter is defined as

$$
G_{\mathbf{v}}=\sqrt{2} G_{\mathrm{F}} \int_{0}^{\infty} \frac{E_{\nu}^{2} \mathrm{~d} E_{\nu}}{(2 \pi)^{3}}\left[f_{\nu_{e}}(\mathbf{p})-f_{\bar{\nu}_{e}}(\mathbf{p})\right],
$$

where $E_{\nu}, \mathbf{p}$, and $\mathbf{v}$ are the neutrino energy, momentum, and velocity, respectively, and $f_{\nu}$ 's are the neutrino occupation numbers. Here, $G_{\mathrm{F}}$ is the Fermi constant. Note that this definition is useful only when $f_{\nu_{\mu, \tau}}=f_{\bar{\nu}_{\mu, \tau}}$. In what follows, we consider the $\phi_{\nu}$-integrated distribution of ELN

$$
G(\mu)=\int_{0}^{2 \pi} \mathrm{d} \phi_{\nu} G_{\mathbf{v}}
$$

where $\mu=\cos \theta_{\nu}$, and $\theta_{\nu}$ and $\phi_{\nu}$ are the zenith and azimuthal angles of the neutrino momentum. Note that the integration over $\phi_{\nu}$ will not generate any fake crossing, though it might erase some of the ELN crossings occurring in $\phi_{\nu}$. We say that $G(\mu)$ has a crossing if it changes its sign at some values of $\mu$.

In the M1 closure scheme, instead of the detailed ELN angular distributions one has access to a few of its moments, defined as

$$
I_{n}=\int_{-1}^{1} \mathrm{~d} \mu \mu^{n} G(\mu) .
$$

To be specific, apart from the evolutions of $I_{0}$ and $I_{1}$ which are computed numerically, one has also access to $I_{2}$ and $I_{3}$ through analytical closure relations. Even with such a limited amount of information, the presence of ELN crossing(s) can still be efficiently evaluated as discussed recently in Ref. [28]. Here it was shown that ELN crossing(s) are guaranteed to occur at a given SN zone if there exists a positive function $\mathcal{F}(\mu)$, for which

$$
I_{\mathcal{F}} I_{0}<0
$$

where

$$
I_{\mathcal{F}}=\int_{-1}^{1} \mathrm{~d} \mu \mathcal{F}(\mu) G(\mu) .
$$

Because the neutrino angular information is provided in terms of $I_{n}$ 's, we choose $\mathcal{F}(\mu)$ to be a polynomial of $\mu$ 


$$
\mathcal{F}(\mu)=\sum_{n=0}^{N} a_{n} \mu^{n}
$$

where $N$ is the highest neutrino angular moment available from numerical simulations and $a_{n}$ 's are arbitrary coefficients for which $\mathcal{F}(\mu)>0$. $I_{\mathcal{F}}$ can then be written in terms of $I_{n}$ 's

$$
I_{\mathcal{F}}=a_{0} I_{0}+a_{1} I_{1}+\cdots+a_{N} I_{N} .
$$

In order to assess the presence of fast instabilities in realistic SN simulation data, for a number of representative post bounce time snapshots and each $\mathrm{SN}$ point, identified by the coordinates $(r, \Theta, \Phi)$, we do the following:

(1) We extract the moments of the ELN up to $I_{3}$, where $I_{2}$ and $I_{3}$ are obtained from $I_{0}$ and $I_{1}$ via Minerbo analytical closure relations (see Eqs. (28a) and (33) in Ref. [60] and Ref. [62]).

(2) We perform a scan of all appropriate quadratic and cubic polynomial functional forms of $\mathcal{F}(\mu)$, i.e., for $a_{3}=0$ and $a_{3} \neq 0$. If we find a polynomial such that Eq. (5) is satisfied, we mark the SN point and we go back to step 1, choosing a different point.

(3) If not, we apply a functional minimization routine to the product $I_{0} I_{\mathcal{F}}$.

(4) We repeat the previous steps around the minimum if it is negative.

The search done with a cubic polynomial is intrinsically more powerful because it can capture finer angular structures and, therefore, narrower ELN crossings. Nevertheless, we find that most of the crossings can be found also with a quadratic polynomial. This is important since it allows us to do not completely rely on $I_{3}$ which is not as accurate as the lower moments [60].

The CCSN data we are about to study were also recently investigated in Ref. [48] where only a number of ELN crossings were found in the convective layer inside the PNS. In that study, the method proposed in Ref. [63] was used, which is based on the instability of a particular Fourier mode (the so-called zeroth mode). However as discussed in the next section, in the same CCSN simulations we find a much larger number of ELN crossings in the SN region outside the PNS. We have also checked that our method can capture the same ELN crossings inside the PNS obtained in Ref. [48]. This is a confirmation of the power of our method, which can also provide a unique insight on the type of the ELN crossing based on the shape of the appropriate $\mathcal{F}(\mu)$ 's.

As a final remark, we emphasize that our method might not capture all the ELN crossings. Indeed, the narrower they are the higher the order of the moments required, i.e., $N>3$. For example, by comparing the number of crossings seen directly with angular distributions and the one found using the moments method with $N=3$, in Ref. [28] it was estimated that almost $50 \%$ of crossings are missed when the moments method is employed. However, in Ref. [28] the moments are calculated directly from the angular distributions, so such an estimate might not be directly applicable to our case.

\section{FAST INSTABILITIES IN 9M ${ }_{\odot}$ AND 20M ${ }_{\odot}$ MODELS}

In this section, we present the results of our ELN crossing search for a number of representative time snapshots, namely $t=100,200,300,400$ and $500 \mathrm{~ms}$ after core bounce for each model. For the sake of plotting, in this section we consider the ELN crossings captured when $a_{3} \neq 0$ though as mentioned previously, the results do not change notably when $a_{3}=0$. Furthermore, in what follows we focus on the ELN crossings in the region outside the PNS to avoid any repetition of the results already discussed in Ref. [48].

\section{A. $9 \mathrm{M}_{\odot}$ supernova model}

\section{1. $t=100 \mathrm{~ms}$}

At this time, the $\mathrm{SN}$ has not exploded yet and it is in the accretion phase where the radius of the $\nu_{e}$ neutrinosphere is $\sim 50 \mathrm{~km}$ and the shock radius is $\sim 150 \mathrm{~km}$. We find ELN crossings starting from $r=91 \mathrm{~km}$ (for which $\sqrt{2} G_{\mathrm{F}} n_{\nu_{e}}=6.9 \times 10^{4} \mathrm{~km}^{-1}$ ) up to distances much larger than the shock radius.

In Fig. 1, we show the shapes of $\mathcal{F}(\mu)$ which allow for capturing the ELN crossings, at two representative radial distances. At $r=100 \mathrm{~km}$ (top panel), $\mathcal{F}(\mu)$ takes large values for $\mu \rightarrow 1$, which implies that the actual ELN angular distributions are most likely negative there and the crossings are in the forward direction ${ }^{1}$. These are indeed the crossings that one expects within/above the neutrino decoupling region. However, this changes at larger radii $(r=206 \mathrm{~km}$, bottom panel $)$ and specifically above the shock. In the preshock region, the ELN crossings are expected to be in the backward direction as discussed before. Hence, $\mathcal{F}(\mu)$ must be large in the backward direction to be capable of capturing the crossings.

The spatial distributions of the ELN crossings within/ above the neutrino decoupling region are strongly correlated with the maxima of $\alpha$, as clearly shown in its Aitoff projection at $r=100 \mathrm{~km}$ reported in Fig. 2. Here, for the sake of presentation, we have shown all the ELN crossings captured between the $\nu_{e}$ neutrinosphere and the shock. We also noted that the distribution of $\alpha$ doest not change noticeably in this range. However, this changes for the backward crossings above the shock $(r>150 \mathrm{~km})$ which are basically ubiquitous [43] (not presented here).

\footnotetext{
${ }^{1}$ In order to capture the ELN crossings, $\mathcal{F}(\mu)$ should be relatively large at $\mu$ 's where the sign of $G(\mu)$ is opposite to the sign of $I_{0}[28]$.
} 

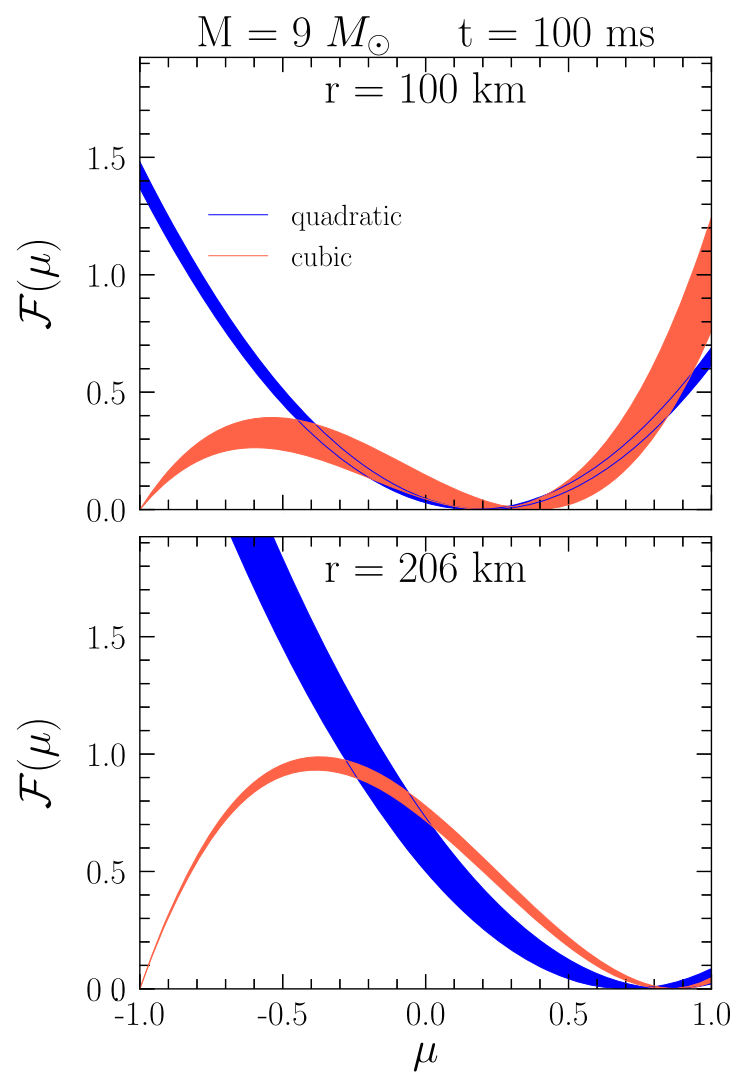

FIG. 1. Shapes of all $\mathcal{F}(\mu)$ 's (at different spatial $\Theta$ 's and $\Phi$ 's) that satisfy Eq. (5) for $9 \mathrm{M}_{\odot}$ model at $t=100 \mathrm{~ms}$, and at $r=$ $100 \mathrm{~km}$ (below the shock) and $206 \mathrm{~km}$ (above the shock), respectively. Red and blue bands represent cubic and quadratic polynomial functions, respectively. The nature of the crossings changes around the shock, which is at $r \simeq 150 \mathrm{~km}$.

\section{2. $t=200 \mathrm{~ms}$}

The model has not exploded yet and the radius of the $\nu_{e}$ neutrinosphere is $\sim 40 \mathrm{~km}$ and the shock radius is $\sim 200 \mathrm{~km}$. In this time snapshot, we find ELN crossings starting from $r=136 \mathrm{~km}$ (for which $\sqrt{2} G_{\mathrm{F}} n_{\nu_{e}}=$ $1.2 \times 10^{4} \mathrm{~km}^{-1}$ ) up to distances much larger than the shock radius. Crossings below the shock are again probably in the forward direction (though assessment is not very easy in this case) and they occur in a very specific spatial direction where $\alpha$ is maximal. In addition, the spatial distribution of the backward ELN crossings is ubiquitous above the shock, as expected.

\section{3. $t=300 \mathrm{~ms}$}

We find no crossings below the shock wave $(r<260 \mathrm{~km})$, whereas the ubiquitous backward crossings above the shock start from about $300 \mathrm{~km}$. The absence of the forward ELN crossings in this time snapshot might be understood by noting that as time goes by, the existing ELN crossings in the neutrino decoupling region gets narrower.

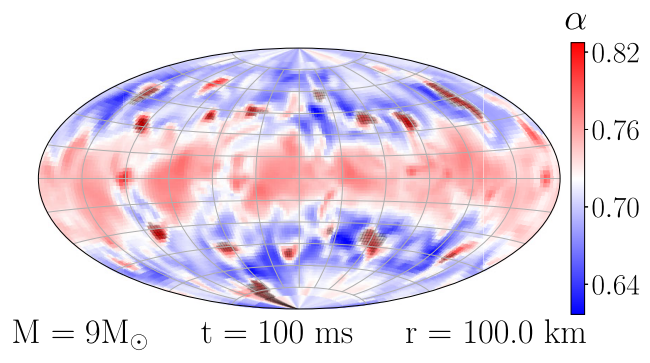

FIG. 2. Aitoff projection of $\alpha$ in the $t=100 \mathrm{~ms}$ snapshot at $r=100 \mathrm{~km}$. The shaded zones indicate the angular coordinates for which we find ELN crossings in the range $[90,150] \mathrm{km}$. Note that the crossings occur where $\alpha$ is maximal. This is a hint in favor of a correlation with the asymmetric PNS convection.

This means that one should have access to higher neutrino angular moments to be able to capture the crossings.

\section{4. $t=400 \mathrm{~ms}$}

At this time, the explosion has already set in with the shock radius being at $\sim 1100 \mathrm{~km}$. The ELN crossings exist below the shock, from $r=56 \mathrm{~km}$ (for which $\sqrt{2} G_{\mathrm{F}} n_{\nu_{e}}=5.1 \times 10^{4} \mathrm{~km}^{-1}$ ) up to $r=1500 \mathrm{~km}$, which is beyond the shock and where we stop our analysis. It seems that in this case only "backward" crossings have been captured, as can be seen from the upper right panel of Fig. 3. However, it should be noted that this type of backward crossings is very different from the one already found in the $t=100-300 \mathrm{~ms}$ snapshots. These crossings occur at smaller radii and more precisely, in the SN postshock region. Hence, they should be mainly attributed to the scattering on free nucleons rather than heavy nuclei, which are already disintegrated almost completely in the SN post-shock region. In particular, these crossings tend to occur in the SN zones where the $\bar{\nu}_{e}\left(\nu_{e}\right)$ angular distribution is more (less) isotropic. This is indicated in Fig. 4, which shows the Aitoff projections of $\tilde{I}_{0} / \tilde{I}_{1}$ for $\nu_{e}$ and $\bar{\nu}_{e}$, where

$$
\tilde{I}_{n}=\sqrt{2} G_{\mathrm{F}} \int_{-1}^{1} \mathrm{~d} \mu \mu^{n} \int_{0}^{\infty} \int_{0}^{2 \pi} \frac{E_{\nu}^{2} \mathrm{~d} E_{\nu} \mathrm{d} \phi_{\nu}}{(2 \pi)^{3}} f_{\nu}(\mathbf{p}) .
$$

This quantity is basically a measure of the isotropy of the neutrino angular distribution: $\tilde{I}_{0} / \tilde{I}_{1}=1$ when the distribution is perfectly peaked in the forward direction, whereas $\tilde{I}_{0} / \tilde{I}_{1}=\infty$ when it is completely isotropic. Note that $\tilde{I}_{n}$ and $I_{n}$ are related by $I_{n}=\left(\tilde{I}_{n}\right)_{\nu_{e}}-\left(\tilde{I}_{n}\right)_{\bar{\nu}_{e}}$. For the locations where these crossings are observed, antineutrinos have a larger $\tilde{I}_{0} / \tilde{I}_{1}$, meaning that they are slightly more isotropic.

Somewhat surprisingly, these backward ELN crossings show strong correlation with the electron fraction, $Y_{e}$, i.e., they occur (or at least they are easier to find) where $Y_{e}$ is maximal, as shown in the middle panels of Fig. 3. One can then also understand the correlation of these crossings with $\alpha$, i.e., they occur where $\alpha$ is minimal: In the region where $\alpha$ 

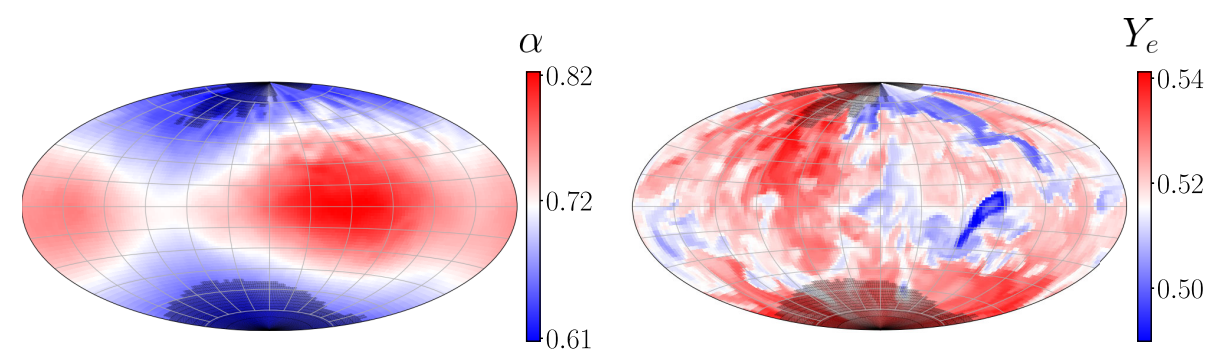

$$
\mathrm{M}=9 \mathrm{M}_{\odot} \quad \mathrm{t}=400 \mathrm{~ms} \quad \mathrm{r}=65.0 \mathrm{~km}
$$
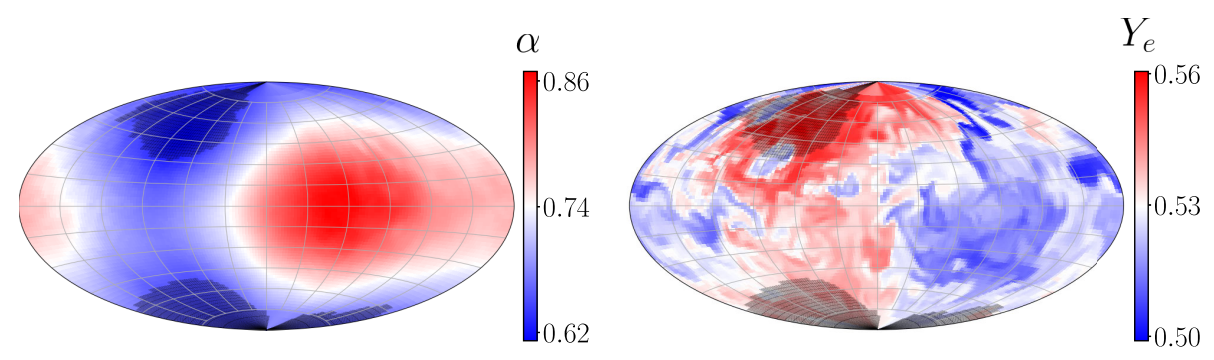

$$
\mathrm{M}=9 \mathrm{M}_{\odot} \quad \mathrm{t}=500 \mathrm{~ms} \quad \mathrm{r}=65.0 \mathrm{~km}
$$
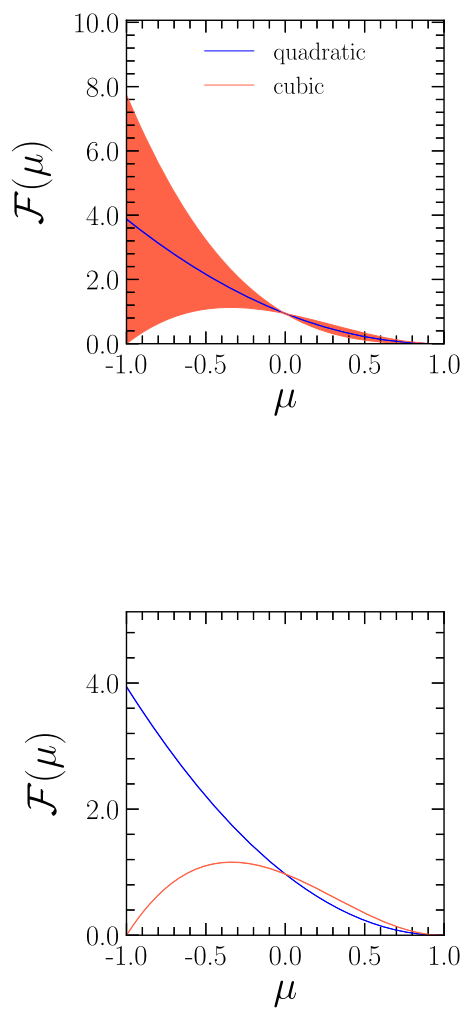

FIG. 3. Left and middle panels: Aitoff projection of $\alpha$ and $Y_{e}$ where the shaded areas represent the SN zones with ELN crossings in the range $[50,150] \mathrm{km}$. Right panels: The shapes of $\mathcal{F}(\mu)$ that satisfy Eq. (5). All the panels are for the $9 \mathrm{M}_{\odot}$ model at $r=65 \mathrm{~km}$, and the upper and lower panels show the data from the $t=400$ and $500 \mathrm{~ms}$ snapshots, respectively. Note that the crossings occur at locations where $\alpha$ has nearly its minimal value, in contrast to what we found for forward crossings. The pattern of $\alpha$ (and $Y_{e}$ ) is a consequence of LESA [51].
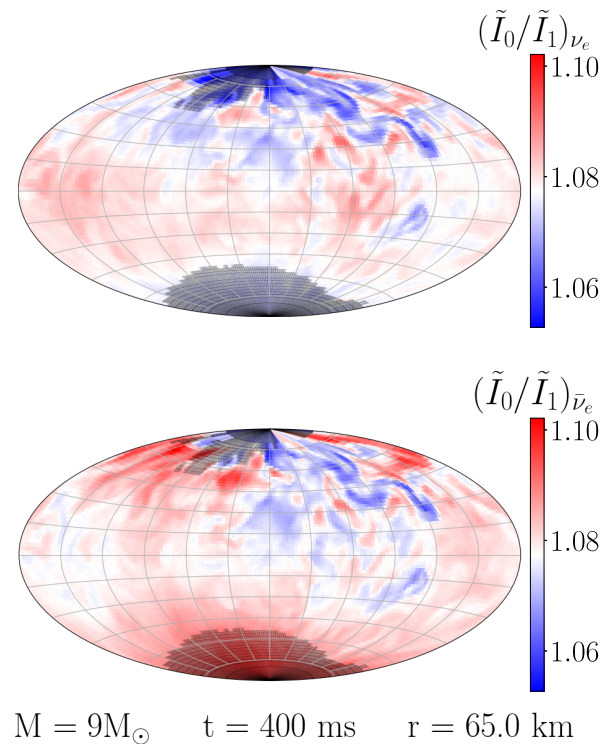

FIG. 4. Aitoff projections of $\tilde{I}_{0} / \tilde{I}_{1}$ for $\nu_{e}$ (upper panel) and $\bar{\nu}_{e}$ (lower panel). Note that $\left(\tilde{I}_{0} / \tilde{I}_{1}\right)$ is larger for $\bar{\nu}_{e}$ at the points where the ELN crossings occur (indicated by shaded areas), suggesting that $\bar{\nu}_{e}$ undergoes more scatterings there. is minimal (due to LESA), $\nu_{e}$ absorption is more dominant, thus driving the ejecta to higher $Y_{e}$. Although making strong conclusions on the exact underlying physics of this phenomenon is extremely difficult, a plausible explanation could be that in the SN zones where $Y_{e}$ is maximal, $\bar{\nu}_{e}\left(\nu_{e}\right)$ is decoupled at larger (smaller) radii due to stronger (weaker) charged-current interactions. Moreover, the ratio of the neutral-current cross sections of neutrinos and antineutrinos is slightly larger when the target particles are protons due to the weak-magnetism corrections [64]. Both of these will result in a $\bar{\nu}_{e}\left(\nu_{e}\right)$ angular distribution which is more (less) isotropic in the $\mathrm{SN}$ zones where $Y_{e}$ is maximal. It is illuminating to note that there is indeed a competition between two effects here. While having smaller values of $\alpha$ reduces the chance of crossings [46,49], the more isotropic neutrino angular distributions (of $\bar{\nu}_{e}$ ) enhance it. Here, it is the latter that apparently wins the competition (though there is no quantitative way to describe this competition).

The reason why this type of crossings is missing in previous time snapshots is that the explosion sets in only at about $300 \mathrm{~ms}$. In non-exploding models and before the explosion sets in, the density gradient around the PNS is 
more shallow. Therefore the decoupling radii of $\nu_{e}$ and $\bar{\nu}_{e}$ are at larger values and also have a larger separation between each other. This leads to relatively large differences of the angular distributions of the neutrinos at given radius exterior to the decoupling region and thus to a reduced relative importance of nucleon scattering between the PNS and the shock. Therefore, nucleon scattering cannot overrule the stronger intrinsic backward component of the neutrino distribution. After the explosion has set in, the density gradient between the PNS and the shock steepens, because the density behind the shock declines, and the neutrinospheres of $\nu_{e}$ and $\bar{\nu}_{e}$ move closer together. In this case, at exterior radii, the $\nu_{e}$ and $\bar{\nu}_{e}$ angular distributions as emitted from their neutrinospheres are less dissimilar, and the nucleon scatterings and charged-current absorptions can affect the ELN distribution relatively more strongly such that backward crossings can occur.

\section{5. $t=500 \mathrm{~ms}$}

In this snapshot, we find ELN crossings starting from $r=68 \mathrm{~km}$ (for which $\sqrt{2} G_{\mathrm{F}} n_{\nu_{e}}=2.6 \times 10^{4} \mathrm{~km}^{-1}$ ) up to $r=1500 \mathrm{~km}$, where we stop our analysis. As indicated in the lower panels of Fig. 3, we again can only capture "backward" crossings which show anti-correlations with $\alpha$ distribution below the shock, analogously to what happens at $t=400 \mathrm{~ms}$.

\section{B. $20 \mathrm{M}_{\odot}$ supernova model}

\section{1. $t=100 \mathrm{~ms}$}

In this time snapshot, we find ELN crossings starting from $r=105 \mathrm{~km}$ (for which $\sqrt{2} G_{\mathrm{F}} n_{\nu_{e}}=1.1 \times 10^{5} \mathrm{~km}^{-1}$ ) up to $r=1500 \mathrm{~km}$, where we stop our analysis. While at lower radial distances we capture forward crossings, at larger radii (above the shock which is at $\sim 150 \mathrm{~km}$ ), the captured crossings are in the backward direction as expected.

\section{2. $t=200-500 \mathrm{~ms}$}

In these time snapshots, we find no ELN crossing below the shock, though the usual backward ubiquitous ELN crossings above the shock (starting from $\simeq 300 \mathrm{~km}$ ) can be observed. This is different from what we observed in the $9 \mathrm{M}_{\odot}$ model. The failure of explosion has indeed important consequences for the occurrence of ELN crossings. As a matter of fact, $\tilde{I}_{0} / \tilde{I}_{1}$ is relatively large for both neutrinos and antineutrinos in the post-shock layer of the $20 \mathrm{M}_{\odot}$ model, as shown in Fig. 6. This comes from the fact that the shock in this nonexploding model retracts after $\sim 100 \mathrm{~ms}$ post bounce. Therefore, on the one hand the post-shock region is (on average) closer to the neutrinospheres, where the neutrino distributions are more isotropic. On the other hand, neutrinos and antineutrinos experience more scatterings which means that their angular distributions become

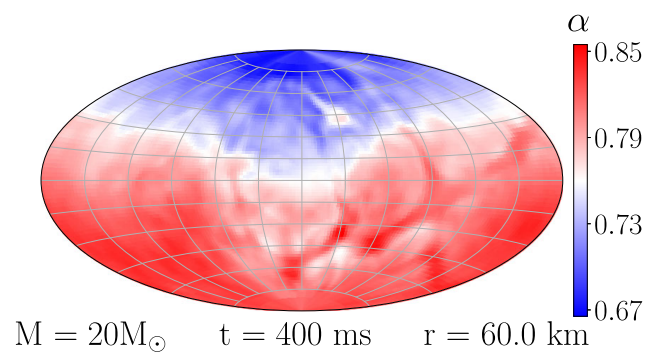

FIG. 5. Aitoff projection of $\alpha$ for the $20 \mathrm{M}_{\odot}$ model in the $t=400 \mathrm{~ms}$ snapshot at $r=60 \mathrm{~km}$. We observe that the average value of $\alpha$ is larger than the one in the $9 \mathrm{M}_{\odot}$ model.

more similar, reducing the chance for the occurrence of ELN crossings. This demonstrates that a large value of $\alpha$ alone (Fig. 5) is not sufficient for the occurrence of forward crossings, but $\tilde{I}_{0} / \tilde{I}_{1}$ also contains crucial information. The high matter density also suppresses backward crossings in the post-shock region of this model as discussed previously, i.e., the intrinsic population of $\nu_{e}$ in the backward direction is so large that scattering-induced backward traveling $\bar{\nu}_{e}$ cannot generate an ELN crossing.

\section{LINEAR STABILITY ANALYSIS}

Until now, we have only focused on the occurrence of ELN crossings in the SN environment. Here we consider flavor instabilities associated with the backward ELN crossings and we discuss some of their important characteristics.

The flavor content of a neutrino gas at each space-time point and in the momentum mode $\mathbf{p}$, can be determined by its flavor density matrix $\varrho_{\mathbf{p}}(t, \mathbf{r})[65]$

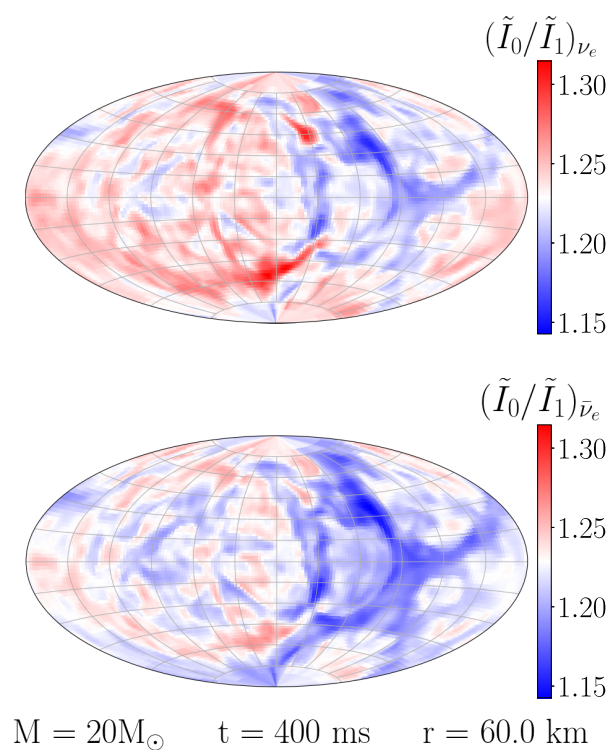

FIG. 6. Aitoff projections of $\tilde{I}_{0} / \tilde{I}_{1}$ for the $20 \mathrm{M}_{\odot}$ model in the $t=400 \mathrm{~ms}$ snapshot at $r=60 \mathrm{~km}$, for $\nu_{e}$ (upper panel) and $\bar{\nu}_{e}$ (lower panel). 


$$
\varrho=\frac{f_{\nu_{e}}+f_{\nu_{x}}}{2}+\frac{f_{\nu_{e}}-f_{\nu_{x}}}{2}\left[\begin{array}{cc}
s & S \\
S^{*} & -s
\end{array}\right]
$$

where the complex and real scalar fields $S$ and $s$ describe the flavor coherence and flavor conversion of neutrinos, respectively. One can similarly define the flavor density matrix, $\bar{Q}$, of antineutrinos. In the linear regime, where significant flavor conversions have not occurred yet $\left(\left|S_{\mathbf{v}}\right| \ll 1\right.$ and $\left.s \approx 1\right)$, the evolution of the flavor coherence term is governed by $[15,66,67]$

$i\left(\partial_{t}+\mathbf{v} \cdot \nabla\right) S_{\mathbf{v}}=\left(\epsilon_{0}+\mathbf{v} \cdot \boldsymbol{\epsilon}\right) S_{\mathbf{v}}-\int \mathrm{d} \Gamma_{\mathbf{v}^{\prime}}\left(1-\mathbf{v} \cdot \mathbf{v}^{\prime}\right) G_{\mathbf{v}^{\prime}} S_{\mathbf{v}^{\prime}}$,

where $\mathrm{d} \Gamma_{\mathbf{v}^{\prime}}$ is the differential solid angle in the direction of $\mathbf{v}^{\prime}, \epsilon_{0}=\lambda+\int \mathrm{d} \Gamma_{\mathbf{v}^{\prime}} G_{\mathbf{v}^{\prime}}$, and $\boldsymbol{\epsilon}=\int \mathrm{d} \Gamma_{\mathbf{v}^{\prime}} G_{\mathbf{v}^{\prime}} \mathbf{v}^{\prime}$. Here we have ignored the vacuum term in the Hamiltonian,

Given the fact that Eq. (11) is a linear equation of motion, it has solutions of the form $S_{\mathbf{v}}(t, \mathbf{r})=Q_{\mathbf{v}} e^{-i \Omega t+i \mathbf{K} \cdot \mathbf{r}}$, where $\Omega$ and $\mathbf{K}$ are independent of $\mathbf{v}$. The neutrino gas is then unstable in the flavor space $\left(S_{\mathbf{v}}\right.$ can grow exponentially) if Eq. (11) features temporal instabilities, i.e., for some real $\mathbf{K}$ there exists a complex $\Omega$ which has a positive imaginary component, namely $\Omega_{\mathrm{i}}=\operatorname{Im}(\Omega)>0$. Such unstable modes with fast growing amplitudes can exist when $G_{\mathbf{v}}$ crosses 0 at some angle(s) $[15,17,18,21,23,39]$.

Although ELN crossings in the backward direction can be extremely narrow, their corresponding fast growth rates are not necessarily small. This is illustrated in the upper panel of Fig. 7, where the growth rate of temporal fast instabilities, $\Omega_{\mathrm{i}}$, is shown for a schematic ELN distribution, defined as

$$
G(\mu)=\xi \times \begin{cases}+1 & \text { for } \mu>\mu_{c} \\ -\delta & \text { for } \mu<\mu_{c}\end{cases}
$$

Here, $\mu_{c}$ is the point where the crossing occurs, $\delta$ is the depth of the backward scattering, and $\xi$ determines the overall scale of the ELN distribution. In our calculation we considered $\mu_{c}=0.95$ and $\delta=10^{-4}$ and $10^{-5}$, which is appropriate for the backward ELN crossings at large radii. The presented growth rates correspond to the temporal unstable modes propagating in the radial direction, assuming that the axial symmetry is preserved in the neutrino gas.

Despite the possibility of having significant growth rates, one should be very careful when interpreting the fast instabilities corresponding to the backward ELN crossings. An extremely important issue, which has been completely overlooked in the literature, is associated with the shape of the angular distribution of the eigenvectors of unstable modes. As indicated in the lower panel of Fig. 7, the eigenvectors of the unstable fast modes are extremely peaked in the backward direction, where the population
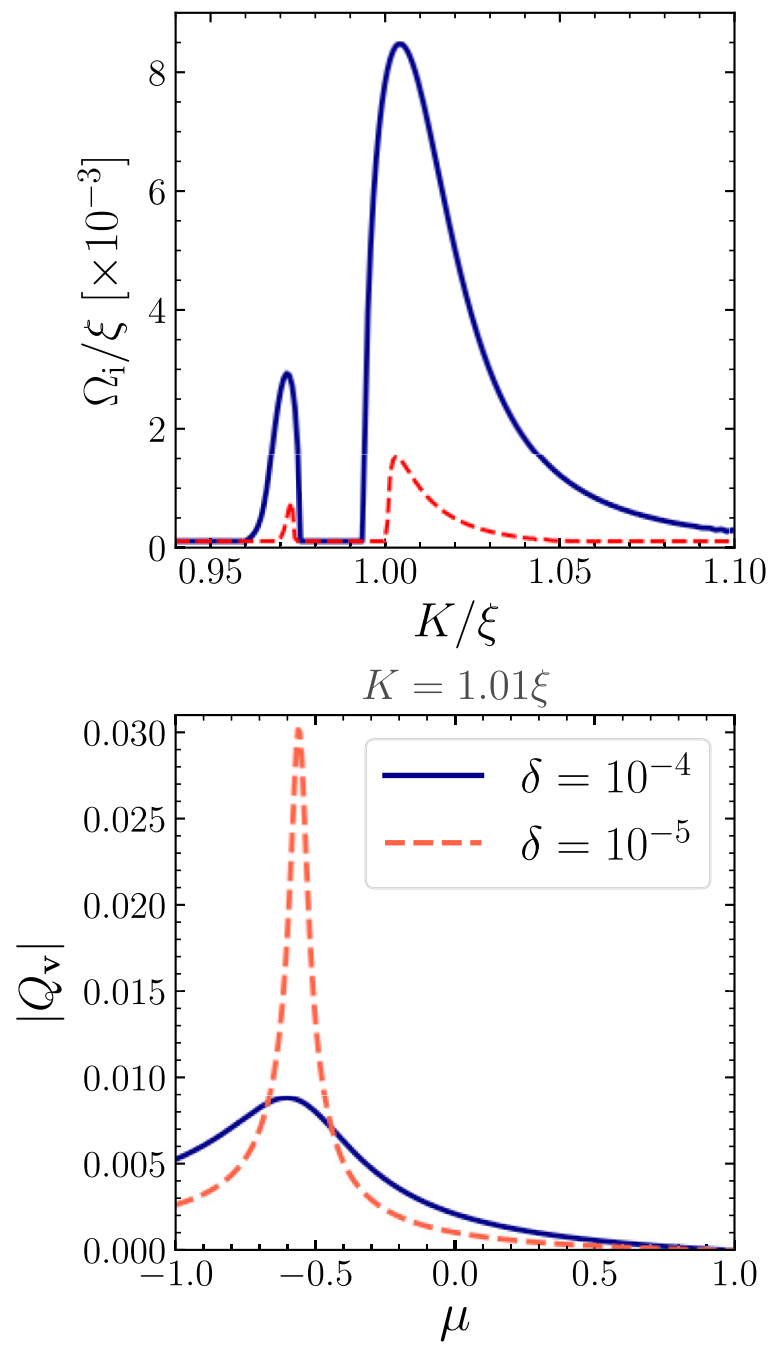

FIG. 7. Upper panel: growth rate of temporal fast instabilities as functions of the real wave number $K$ of the fast modes propagating in the radial direction. Note that despite the fact that the crossings in the backward direction are very shallow, the growth rates can still be significant for large neutrino number densities. For instance, for $\xi=10^{4} \mathrm{~km}^{-1}$ and $\delta=10^{-4}$, the growth rate can be as high as $10^{2} \mathrm{~km}^{-1}$. Lower panel: an example of the angular distributions of the eigenvectors of unstable modes, $Q_{\mathrm{v}}$, which is normalized to one here. These distributions correspond to the unstable modes at $K=1.01 \xi$, where the growth rates are close to their maxima. Note that the amplitude of $Q_{\mathrm{v}}$ is extremely small in the forward direction where the majority of neutrinos are traveling. In these calculations we assumed that the neutrino gas possesses axial symmetry.

of neutrinos is extremely small. This comes as no surprise since the ELN crossings are also generated by this small population. However, this means that in the linear regime, the unstable modes can mostly affect the neutrinos propagating in the backward direction, which are a tiny fraction of all neutrinos. Despite this, significant flavor conversions can still occur in the nonlinear regime. Hence, to have a better understanding of this issue, sophisticated neutrino 
flavor conversion simulations in the nonlinear regime are absolutely indispensable.

\section{DISCUSSION AND CONCLUSIONS}

To assess the occurrence of ELN crossings and their associated fast neutrino flavor instabilities in CCSNe, we have analyzed two recent state-of-the-art 3D CCSN simulations of a $9 \mathrm{M}_{\odot}$ and a $20 \mathrm{M}_{\odot}$ progenitor model, using the method proposed recently in Ref. [28]. Not only do we confirm the results previously reported in the literature using post-processing calculations [44] or 1D/2D models $[43,46]$, but we also highlight some new insights. First, we bring up the possibility of having backward ELN crossings in the SN post-shock region. Second, we argue that the failure of the SN explosion can have important consequences for the existence of ELN crossings, i.e., it can seriously hinder their occurrence in the $\mathrm{SN}$ environment.

In the case of the successfully exploding $9 \mathrm{M}_{\odot} \mathrm{SN}$ model, we observed a large number of ELN crossings in the forward direction in the $100 \mathrm{~ms}$ and $200 \mathrm{~ms}$ snapshots within/above the neutrino decoupling region (see Fig. 2). The spatial distribution of these ELN crossings is correlated with the distribution of $\alpha$, which itself originates from the convective activity inside the PNS (which is thought to generate LESA at later times, namely $t_{\mathrm{pb}} \gtrsim 300 \mathrm{~ms}$ in this model). However, such ELN crossings are not observed in the later snapshots. This may look strange at first, considering the correlation of these crossings with LESA which becomes stronger at later times in this model. This means that one should expect a smaller difference between $n_{\nu_{e}}$ and $n_{\bar{\nu}_{e}}$ (in the hemisphere opposite to the LESA dipole direction) and consequently, a higher chance for the occurrence of forward ELN crossings. Despite this, as time goes by the difference between the radii of the neutrinospheres of $\nu_{e}$ and $\bar{\nu}_{e}$ decreases, making their angular distributions more similar in the forward direction. As a result, the range of $\mu$ where $G(\mu)$ changes its sign becomes extremely narrow. In the context of our method, this means that although forward ELN crossings might be more likely to occur at later times $[44,46]$, higher neutrino angular moments are required to capture them.

We also observe a ubiquitous distribution of ELN crossings in the backward direction in the pre-shock SN region in all time snapshots of the $9 \mathrm{M}_{\odot}$ model as well as the $20 \mathrm{M}_{\odot}$ one. Such ELN crossings, first reported in Ref. [43] in a 1D SN model, are thought to be generated by coherent neutrino scattering on heavy nuclei, which are enhanced for the slightly more energetic $\bar{\nu}_{e}$. Our results confirm the presence of these crossings in multi-D SN models and show that they are probably very generic.

Somewhat surprisingly, we also find backward ELN crossings in the post-shock region of the 400 and $500 \mathrm{~ms}$ snapshots of the $9 \mathrm{M}_{\odot}$ model at radii which can be as small as $\sim 50 \mathrm{~km}$. Unlike the backward ELN crossings in the $\mathrm{SN}$ pre-shock region, which are generated by coherent scatterings on heavy nuclei, the backward crossings below the $\mathrm{SN}$ shock should be attributed to the scatterings on free nucleons since heavy nuclei here are disintegrated almost completely.

A remarkable feature of the backward ELN crossings in the SN post-shock region is that their spatial distribution shows a strong correlation with $Y_{e}$ (which leads to an anticorrelation with $\alpha$ ), in contrast to the forward crossings. Though it is very difficult to draw any conclusion about the exact nature of this pattern, it could be explained, at least partially, by noting that in these $\mathrm{SN}$ zones where $Y_{e}$ is larger, $\bar{\nu}_{e}\left(\nu_{e}\right)$ decouples at larger (smaller) radii due to stronger (weaker) charged-current interactions. Moreover, the neutral-current scattering of $\bar{\nu}_{e}$ on free nucleons is slightly enhanced therein due to the larger fraction of protons. The presence of such backward ELN crossings implies that at least at later times, one could expect an almost ubiquitous distribution of ELN crossings below the SN shock, forward crossings around the minima of $Y_{e}$ and backward crossings around its maxima.

As for the nonexploding $20 \mathrm{M}_{\odot}$ model, in the post-shock region we observe forward crossings only in the $100 \mathrm{~ms}$ snapshot, whereas backward crossings are ubiquitous in the preshock region. As a matter of fact, the failure of the explosion has significant consequences for the occurrence of fast instabilities. Due to the failure of the explosion, the post-shock region is closer to the neutrinospheres where the neutrino angular distributions are more isotropic. In addition, the matter density below the shock increases with time as the shock retracts, which implies more scatterings for $\nu_{e}$ and $\bar{\nu}_{e}$. This means that their angular distributions become more isotropic (and therefore more similar) which in turn reduces the chance for the occurrence of forward ELN crossings. Note that this happens despite the fact that the overall value of $\alpha$ is larger in this case compared to the $9 \mathrm{M}_{\odot}$ SN model. This clearly shows that one needs care when assessing the occurrence of forward ELN crossings by just considering the parameter $\alpha^{2}$.

A failed explosion can similarly explain why there are no backward ELN crossings in the post-shock layer of the $20 \mathrm{M}_{\odot} \mathrm{SN}$ model. Due to the high matter density below the shock and the closer distance to the PNS, the backward direction is still dominated by preexisting $\nu_{e}$ 's, and the higher energetic $\bar{\nu}_{e}$ 's cannot generate an ELN crossing in the backward direction. This is also why the backward ELN crossings in the post-shock region are not observed in the earlier snapshots of the $9 \mathrm{M}_{\odot}$ model where the shock is still relatively close to the PNS and the matter density is high.

Note that this argument only suggests that the large matter densities behind the stalled or retreating shock can hinder the occurrence of ELN crossings. However, one

\footnotetext{
${ }^{2}$ Note that this case is different from the case studied in Ref. [47] where the overall value of $\alpha$ was very small for the nonexploding model.
} 
cannot make any quantitative estimate on the exact level of suppression of the ELN crossings, which depends on a number of factors such as the SN model, its mass, the fate of the explosion, the neutrino transport method, etc. This means that though less probable, ELN crossings can still occur in the post-shock region of nonexploding models. As an example, in the context of 1D models, Ref. [61] found a number of backward ELN crossings in the post-shock region of a nonexploding $20 \mathrm{M}_{\odot}$ model in the presence of muon creation in the SN core, which is not included in the models analyzed herein. Overall, this highlights the importance of more realistic modelings of CCSNe to have a better understanding of the occurrence of ELN crossings and, therefore, of neutrino flavor conversions therein, given the fact that the fate of the explosion may remarkably affect the reliability of our predictions of this phenomenon.

In summary, apart from confirming the previous reports of the occurrence of ELN crossings and fast instabilities by the analysis of our 3D state-of-the-art CCSN simulations, we point out a new possibility for the occurrence of probably scattering-induced ELN crossings in the postshock region of exploding SNe. Nevertheless, it should be kept in mind that the occurrence of fast instabilities does not necessarily lead to (significant) flavor conversions [68]. Our results also suggest that the occurrence of ELN crossings could be strongly hindered in nonexploding SN models with high mass accretion rates. A key limitation of our study is that it is based on only a few of the neutrino angular moments and therefore, our understandings are still somewhat speculative. In particular, drawing strong conclusions from a thus found crossing on the underlying physics is extremely difficult. In order to have a more accurate picture of the physics of fast neutrino flavor conversions in $\mathrm{CCSNe}$, one needs to consider the full phase-space distribution functions of neutrinos. Therefore, 3D CCSN simulations with Boltzmann neutrino transport are indispensable. Moreover, the occurrence of ELN crossings should be studied for a wide range of progenitor masses, which can offer accretion and explosion conditions that are considerably different from those of the considered $9 \mathrm{M}_{\odot}$ and $20 \mathrm{M}_{\odot}$ cases because of the progenitor-specific accretion histories after core bounce.

\section{ACKNOWLEDGMENTS}

We would like to thank Georg Raffelt for his comments on the manuscript and useful discussions. We acknowledge partial support by the Deutsche Forschungsgemeinschaft (DFG, German Research Foundation) through Grant Sonderforschungsbereich (Collaborative Research Center) SFB1258 "Neutrinos and Dark Matter in Astroand Particle Physics (NDM)". Additionally, F. C.'s work at Virginia Tech is supported by the U.S. Department of Energy under the Awards No. DE-SC0020250 and No. DESC0020262 and at Garching, funding by the European Research Council through Grant ERC-AdG No. 341157COCO2CASA and by DFG under Germany's Excellence Strategy through Cluster of Excellence ORIGINS (EXC2094) - 390783311 is acknowledged. Computer resources for this project have been provided by the Leibniz Supercomputing Centre (LRZ) under LRZ project ID pr62za and by the Max Planck Computing and Data Facility (MPCDF) on the HPC system Hydra. I. T. acknowledges support from the Villum Foundation (Project No. 13164), the Danmarks Frie Forskningsfonds (Project No. 8049-00038B) and the Knud $\mathrm{H} \varnothing$ jgaard Foundation.
[1] H.-T. Janka, Explosion mechanisms of core-collapse supernovae, Annu. Rev. Nucl. Part. Sci. 62, 407 (2012).

[2] A. Burrows, Colloquium: Perspectives on core-collapse supernova theory, Rev. Mod. Phys. 85, 245 (2013).

[3] A. Mezzacappa, Ascertaining the core collapse supernova mechanism: The state of the art and the road ahead, Annu. Rev. Nucl. Part. Sci. 55, 467 (2005).

[4] S. Pastor and G. Raffelt, Flavor Oscillations in the Supernova Hot Bubble Region: Nonlinear Effects of Neutrino Background, Phys. Rev. Lett. 89, 191101 (2002).

[5] H. Duan, G. M. Fuller, J. Carlson, and Y.-Z. Qian, Simulation of coherent non-linear neutrino flavor transformation in the supernova environment. 1. Correlated neutrino trajectories, Phys. Rev. D 74, 105014 (2006).

[6] H. Duan, G. M. Fuller, and Y.-Z. Qian, Collective neutrino oscillations, Annu. Rev. Nucl. Part. Sci. 60, 569 (2010).
[7] Y.Z. Qian and S. E. Woosley, Nucleosynthesis in neutrino driven winds: 1. The physical conditions, Astrophys. J. 471, 331 (1996).

[8] A. Mirizzi, I. Tamborra, H.-T. Janka, N. Saviano, K. Scholberg, R. Bollig, L. Hudepohl, and S. Chakraborty, Supernova neutrinos: Production, oscillations and detection, Riv. Nuovo Cimento 39, 1 (2016).

[9] K. Scholberg, Supernova neutrino detection, Annu. Rev. Nucl. Part. Sci. 62, 81 (2012).

[10] C. Lunardini, Diffuse supernova neutrinos at underground laboratories, Astropart. Phys. 79, 49 (2016).

[11] J. F. Beacom, The diffuse supernova neutrino background, Annu. Rev. Nucl. Part. Sci. 60, 439 (2010).

[12] R. F. Sawyer, Speed-up of neutrino transformations in a supernova environment, Phys. Rev. D 72, 045003 (2005).

[13] R. F. Sawyer, Neutrino Cloud Instabilities Just Above the Neutrino Sphere of a Supernova, Phys. Rev. Lett. 116, 081101 (2016). 
[14] S. Chakraborty, R. S. Hansen, I. Izaguirre, and G. Raffelt, Self-induced neutrino flavor conversion without flavor mixing, J. Cosmol. Astropart. Phys. 03 (2016) 042.

[15] I. Izaguirre, G. Raffelt, and I. Tamborra, Fast Pairwise Conversion of Supernova Neutrinos: A Dispersion-Relation Approach, Phys. Rev. Lett. 118, 021101 (2017).

[16] M.-R. Wu and I. Tamborra, Fast neutrino conversions: Ubiquitous in compact binary merger remnants, Phys. Rev. D 95, 103007 (2017).

[17] F. Capozzi, B. Dasgupta, E. Lisi, A. Marrone, and A. Mirizzi, Fast flavor conversions of supernova neutrinos: Classifying instabilities via dispersion relations, Phys. Rev. D 96, 043016 (2017).

[18] S. Abbar and H. Duan, Fast neutrino flavor conversion: Roles of dense matter and spectrum crossing, Phys. Rev. D 98, 043014 (2018).

[19] S. Abbar and M. C. Volpe, On fast neutrino flavor conversion modes in the nonlinear regime, Phys. Lett. B 790 , 545 (2019).

[20] F. Capozzi, B. Dasgupta, A. Mirizzi, M. Sen, and G. Sigl, Collisional Triggering of Fast Flavor Conversions of Supernova Neutrinos, Phys. Rev. Lett. 122, 091101 (2019).

[21] C. Yi, L. Ma, J. D. Martin, and H. Duan, Dispersion relation of the fast neutrino oscillation wave, Phys. Rev. D 99, 063005 (2019).

[22] J. D. Martin, C. Yi, and H. Duan, Dynamic fast flavor oscillation waves in dense neutrino gases, Phys. Lett. B 800, 135088 (2020).

[23] F. Capozzi, G. Raffelt, and T. Stirner, Fast neutrino flavor conversion: Collective motion vs decoherence, J. Cosmol. Astropart. Phys. 09 (2019) 002.

[24] L. Johns, H. Nagakura, G. M. Fuller, and A. Burrows, Neutrino oscillations in supernovae: Angular moments and fast instabilities, Phys. Rev. D 101, 043009 (2020).

[25] S. Shalgar, I. Padilla-Gay, and I. Tamborra, Neutrino propagation hinders fast pairwise flavor conversions, J. Cosmol. Astropart. Phys. 06 (2020) 048.

[26] J. F. Cherry, G. M. Fuller, S. Horiuchi, K. Kotake, T. Takiwaki, and T. Fischer, Time of flight and supernova progenitor effects on the neutrino halo, Phys. Rev. D 102, 023022 (2020).

[27] M. Chakraborty and S. Chakraborty, Three flavor neutrino conversions in supernovae: Slow and fast instabilities, J. Cosmol. Astropart. Phys. 01 (2020) 005.

[28] S. Abbar, Searching for fast neutrino flavor conversion modes in core-collapse supernova simulations, J. Cosmol. Astropart. Phys. 05 (2020) 027.

[29] F. Capozzi, M. Chakraborty, S. Chakraborty, and M. Sen, Fast Flavor Conversions in Supernovae: The Rise of mu-tau Neutrinos, Phys. Rev. Lett. 125, 251801 (2020).

[30] Z. Xiong, A. Sieverding, M. Sen, and Y.-Z. Qian, Potential impact of fast flavor oscillations on neutrino-driven winds and their nucleosynthesis, Astrophys. J. 900, 144 (2020).

[31] S. Bhattacharyya and B. Dasgupta, Late-time behavior of fast neutrino oscillations, Phys. Rev. D 102, 063018 (2020).

[32] S. Shalgar and I. Tamborra, Dispelling a myth on dense neutrino media: Fast pairwise conversions depend on energy, J. Cosmol. Astropart. Phys. 01 (2021) 014.

[33] L. Johns, H. Nagakura, G. M. Fuller, and A. Burrows, Fast oscillations, collisionless relaxation, and spurious evolution of supernova neutrino flavor, Phys. Rev. D 102, 103017 (2020).

[34] S. Bhattacharyya and B. Dasgupta, Fast Flavor Depolarization of Supernova Neutrinos, Phys. Rev. Lett. 126, 061302 (2021).

[35] M. George, M.-R. Wu, I. Tamborra, R. Ardevol-Pulpillo, and H.-T. Janka, Fast neutrino flavor conversion, ejecta properties, and nucleosynthesis in newly-formed hypermassive remnants of neutron-star mergers, Phys. Rev. D 102, 103015 (2020).

[36] S. Shalgar and I. Tamborra, A change of direction in pairwise neutrino conversion physics: The effect of collisions, Phys. Rev. D 103, 063002 (2021).

[37] I. Tamborra and S. Shalgar, New developments in flavor evolution of a dense neutrino gas, arXiv:2011.01948.

[38] S. Abbar, Turbulence fingerprint on collective oscillations of supernova neutrinos, Phys. Rev. D 103, 045014 (2021).

[39] B. Dasgupta, A. Mirizzi, and M. Sen, Fast neutrino flavor conversions near the supernova core with realistic flavordependent angular distributions, J. Cosmol. Astropart. Phys. 02 (2017) 019.

[40] R. F. Sawyer, The multi-angle instability in dense neutrino systems, Phys. Rev. D 79, 105003 (2009).

[41] S. Chakraborty, R. Hansen, I. Izaguirre, and G. Raffelt, Collective neutrino flavor conversion: Recent developments, Nucl. Phys. B908, 366 (2016).

[42] I. Tamborra, L. Huedepohl, G. Raffelt, and H.-T. Janka, Flavor-dependent neutrino angular distribution in corecollapse supernovae, Astrophys. J. 839, 132 (2017).

[43] T. Morinaga, H. Nagakura, C. Kato, and S. Yamada, Fast neutrino-flavor conversion in the preshock region of corecollapse supernovae, Phys. Rev. Research 2, 012046 (2020).

[44] S. Abbar, H. Duan, K. Sumiyoshi, T. Takiwaki, and M. C. Volpe, On the occurrence of fast neutrino flavor conversions in multidimensional supernova models, Phys. Rev. D 100, 043004 (2019).

[45] M. Delfan Azari, S. Yamada, T. Morinaga, H. Nagakura, S. Furusawa, A. Harada, H. Okawa, W. Iwakami, and K. Sumiyoshi, Fast collective neutrino oscillations inside the neutrino sphere in core-collapse supernovae, Phys. Rev. D 101, 023018 (2020).

[46] H. Nagakura, T. Morinaga, C. Kato, and S. Yamada, Fastpairwise collective neutrino oscillations associated with asymmetric neutrino emissions in core-collapse supernova, Astrophys. J. 886, 139 (2019).

[47] S. Abbar, H. Duan, K. Sumiyoshi, T. Takiwaki, and M. C. Volpe, Fast neutrino flavor conversion modes in multidimensional core-collapse supernova models: The role of the asymmetric neutrino distributions, Phys. Rev. D 101, 043016 (2020).

[48] R. Glas, H. T. Janka, F. Capozzi, M. Sen, B. Dasgupta, A. Mirizzi, and G. Sigl, Fast neutrino flavor instability in the neutron-star convection layer of three-dimensional supernova models, Phys. Rev. D 101, 063001 (2020).

[49] S. Shalgar and I. Tamborra, On the occurrence of crossings between the angular distributions of electron neutrinos and antineutrinos in the supernova core, Astrophys. J. 883, 80 (2019).

[50] I. Tamborra, F. Hanke, H.-T. Janka, B. Müller, G. G. Raffelt, and A. Marek, Self-sustained asymmetry of lepton-number 
emission: A new phenomenon during the supernova shockaccretion phase in three dimensions, Astrophys. J. 792, 96 (2014).

[51] R. Glas, H. T. Janka, T. Melson, G. Stockinger, and O. Just, Effects of LESA in three-dimensional supernova simulations with multi-dimensional and Ray-by-Ray-plus neutrino transport, Astrophys. J. 881, 36 (2018).

[52] H. Nagakura, K. Sumiyoshi, and S. Yamada, Possible early linear acceleration of proto-neutron stars via asymmetric neutrino emission in core-collapse supernovae, Astrophys. J. Lett. 880, L28 (2019).

[53] W. Iwakami, H. Okawa, H. Nagakura, A. Harada, S. Furusawa, K. Sumiyoshi, H. Matsufuru, and S. Yamada, Simulations of the early postbounce phase of core-collapse supernovae in three-dimensional space with full Boltzmann neutrino transport, Astrophys. J. 903, 82 (2020).

[54] H. Nagakura, W. Iwakami, S. Furusawa, H. Okawa, A. Harada, K. Sumiyoshi, S. Yamada, H. Matsufuru, and A. Imakura, Simulations of core-collapse supernovae in spatial axisymmetry with full Boltzmann neutrino transport, Astrophys. J. 854, 136 (2018).

[55] A. Harada, H. Nagakura, W. Iwakami, H. Okawa, S. Furusawa, H. Matsufuru, K. Sumiyoshi, and S. Yamada, On the neutrino distributions in phase space for the rotating core-collapse supernova simulated with a Boltzmannneutrino-radiation-hydrodynamics code, Astrophys. J. 872, 181 (2019).

[56] A. Harada, H. Nagakura, W. Iwakami, H. Okawa, S. Furusawa, K. Sumiyoshi, H. Matsufuru, and S. Yamada, The Boltzmann-radiation-hydrodynamics simulations of core-collapse supernovae with different equations of state: The role of nuclear composition and the behavior of neutrinos, Astrophys. J. 902, 150 (2020).

[57] C. Chan and B. Müller, A novel multidimensional Boltzmann neutrino transport scheme for core-collapse supernovae, Mon. Not. R. Astron. Soc. 496, 2000 (2020).
[58] R. Glas, O. Just, H. T. Janka, and M. Obergaulinger, Threedimensional core-collapse supernova simulations with multidimensional neutrino transport compared to the Ray-by-Ray-plus approximation, Astrophys. J. 873, 45 (2019).

[59] M. Obergaulinger, P. Cerda-Duran, E. Muller, and M. Aloy, Semi-global simulations of the magneto-rotational instability in core collapse supernovae, Astron. Astrophys. 498, 241 (2009).

[60] O. Just, M. Obergaulinger, and H. Janka, A new multidimensional, energy-dependent two-moment transport code for neutrino-hydrodynamics, Mon. Not. R. Astron. Soc. 453, 3387 (2015).

[61] O. Just, R. Bollig, H.-T. Janka, M. Obergaulinger, R. Glas, and S. Nagataki, Core-collapse supernova simulations in one and two dimensions: comparison of codes and approximations, Mon. Not. R. Astron. Soc. 481, 4786 (2018).

[62] G. N. Minerbo, Maximum entropy eddington factors, J. Quantum Spec. Radiat. Transf. 20, 541 (1978).

[63] B. Dasgupta, A. Mirizzi, and M. Sen, Simple method of diagnosing fast flavor conversions of supernova neutrinos, Phys. Rev. D 98, 103001 (2018).

[64] C. Horowitz, Weak magnetism for anti-neutrinos in supernovae, Phys. Rev. D 65, 043001 (2002).

[65] G. Sigl and G. Raffelt, General kinetic description of relativistic mixed neutrinos, Nucl. Phys. B406, 423 (1993).

[66] A. Banerjee, A. Dighe, and G. Raffelt, Linearized flavorstability analysis of dense neutrino streams, Phys. Rev. D 84, 053013 (2011).

[67] D. Väänänen and C. Volpe, Linearizing neutrino evolution equations including neutrino-antineutrino pairing correlations, Phys. Rev. D 88, 065003 (2013).

[68] I. Padilla-Gay, S. Shalgar, and I. Tamborra, Multidimensional solution of fast neutrino conversions in binary neutron star merger remnants, J. Cosmol. Astropart. Phys. 01 (2021) 017. 